MATEC Web of Conferences 11,02001 (2014)

DOI: $10.1051 /$ matecconf $/ 20141102001$

(C) Owned by the authors, published by EDP Sciences, 2014

\title{
Amélioration de la durabilité des briques de terre comprimée à base d'un sol de la région de Biskra
}

\section{Improved durability of compressed earth bricks based on a soil of the region of Biskra}

\author{
O. Izemmourena ${ }^{1}$, A. Guettala ${ }^{1}$ \\ ${ }^{1}$ Laboratoire de Recherche en Génie Civil " LRGC " Université de Biskra. Alger.
}

\begin{abstract}
Its availability and low cost have made the earth in most parts of the world, one of the most answered and oldest construction materials. The Earth presents an ecological interest where its construction participates well to the environmental protection at the chemical industry (cement production) where at energetic economy (making fired bricks). The problem posed by the traditional use of the earth is a problem of durability of the material itself. The objective of this work is mainly set on improving the characteristics of compressed earth bricks, especially the behavior towards water. The objective of this work is mainly fixed on improving the characteristics of compressed earth bricks, especially insensitivity to water. Stabilization is achieved by granulometric Correction carried out by two types of crushed sand with five levels $0,10,20,30$, $40 \%$ of the weight of the soil by fixing the percentage of cement at $5 \%$ and of the stress compaction at 100 bar. In this work we studied the influence of different levels of crushed sand on the mechanical compressive strengths at dry and wet, sustainability, capillary absorption, total absorption, action freeze-thaw. The results show that increasing the concentration of sand significantly improves the mechanical strength and present optimum $30 \%$ sand for durability tests.
\end{abstract}

Résumé. Sa disponibilité et son faible coût ont fait de la terre dans la plupart des régions du globe, un des matériaux de construction les plus répondus et plus anciens. La terre présente un intérêt écologique évidant, où sa construction participe bien à la protection de l'environnement à l'industrie chimique (fabrication de ciment) où à l'économie energétique (fabrication des briques cuites). Le problème, que pose l'utilisation traditionnelle de la terre, est donc un problème de durabilité du matériau lui-même. L'objectif de ce travail est fixé principalement sur l'amélioration des caractéristiques des briques de terre comprimée, surtout insensibilité à l'eau. La stabilisation est obtenue par correction granulométrique réalisée par deux types de sable concassée avec cinq teneurs $0,10,20,30,40 \%$ du poids du sol, en fixant le dosage en ciment à $5 \%$ et la contrainte de compactage à 100 bars. Dans ce travail on a étudié l'influence des différents teneurs en sable concassée sur les résistances mécaniques de compression à l'état sec et à l'état humide, sur la durabilité, l'absorption capillaire, l'absorption totale, l'action gel-dégel. Les résultats obtenus montrent que l'augmentation de la concentration du sable améliore considérablement les résistances mécaniques et présente un optimum de $30 \%$ de sable pour les essais de durabilité.

\section{Introduction}

Depuis quelques années, on assiste à un ressaut intéressant de la recherche sur les matériaux dits traditionnels. On cherche des logements économiques suite à la rareté et au coût excessif des logements pour les pays en développement notamment l'Algérie où les architectes étaient convaincus que la solution était dans le passé. Néanmoins, le problème qui évoque certaines préoccupations est l'amélioration de sa durabilité lorsqu'il est soumis à des conditions climatiques délétères $[1,2]$.

En effet, la nécessité d'obtenir un matériau de base durable dans les constructions des briques de terre comprimée était à l'origine un facteur favorisant les ouvrages ayant une durée de vie indéfiniment longue à l'exigence d'un minimum d'entretien, doit conduire à envisager un traitement qui, tout en assurant une résistance mécanique suffisante, tend à augmenter son insensibilité à l'eau [2,3]. 
Dans le cadre de cette étude, nous avons entrepris de contribuer à l'étude du B.T.C destiné à la construction. Ce choix résulte à l'intérêt économique et thermique que revêt ce matériau. Trois facteurs essentiels interviennent pour augmenter la qualité des briques de terre comprimée sa composition, la teneur en liant et la compression de compactage. Cette amélioration du comportement du sol peut être obtenue de différentes manières correction granulométrique, addition du liant et compactage. La combinaison entre ces facteurs s'avère plus efficace [4].

Dans ce travaille on a étudié la stabilisation par la correction granulométrique qui faite par deux types de sable concassée avec cinq teneurs: $0,10,20,30,40 \%$ du poids du sol. Afin d'optimiser le sable, nous avons fixé la teneur en ciment à $5 \%$ et la contrainte de compactage à 100 bars. Les résultats obtenus sont présentés sous forme des courbes et des histogrammes, chaque valeur expérimentale est la moyenne des valeurs obtenues sur trois éprouvettes.

\section{Caractéristiques des matériaux utilisés}

\subsection{Sable}

Les sables choisis ont été essentiellement utilisés pour la correction de la granulométrie des terres contenants une fortes proportion d'argile

On a utilisé deux types de sable :

Sable $N^{\circ} 1$ : sable concassée.

Sable $\mathrm{N}^{\circ} 2$ : sable concassée tamisé (sans fines).

\subsubsection{Caractéristiques physiques}

Les résultats des tests sur les sables utilisés (en utilisant les normes Françaises AFNOR 1984 [5] sont présentés sur le tableau 1 .

Tableau 1 : Caractéristiques physiques des sables utilisés

\begin{tabular}{|c|c|c|c|c|c|}
\hline & \multirow{2}{*}{$\begin{array}{c}\text { Type } \\
\text { du } \\
\text { sable }\end{array}$} & $\begin{array}{c}\text { Masse } \\
\text { volumique } \\
\text { spécifique } \\
\gamma \mathrm{s}(\mathrm{g} / \mathrm{cm} 3)\end{array}$ & $\begin{array}{c}\text { Masse } \\
\text { volumique } \\
\text { apparente } \\
\rho 0 \\
(\mathrm{~kg} / \mathrm{cm} 3)\end{array}$ & $\begin{array}{c}\text { porosit } \\
\text { é }\end{array}$ & \multicolumn{2}{|c|}{$\begin{array}{c}\text { Equivalent de } \\
\text { sable E.S }\end{array}$} \\
\cline { 5 - 6 } & 2.64 & 1.52 & 42.42 & 70.42 & 64.08 \\
\hline $\begin{array}{c}\text { Sable } \\
1\end{array}$ & 2.75 & 1.34 & 51.27 & 96.84 & 95.79 \\
\hline $\begin{array}{c}\text { Sable } \\
2\end{array}$ & 2.3 viston \\
\hline
\end{tabular}

\subsection{Sol}

Le sol utilisé dans notre travail trouve son appartenance dans la région de Biskra. L'emploi judicieux des matériaux utilisés dans la construction exige la connaissance de leurs diverses qualités, physiques, chimiques, minéralogiques, physico-chimiques et mécaniques, pour permettre de faire un choix répondant à leur destination.

\subsubsection{Analyse granulométrique}

Les figures 1, 2 présentent la courbe granulométrique du sol ainsi que des mélanges (sol, sable) avec la courbe idéale et le fuseau limite pour les briques de terre stabilisée. L'analyse de la courbe granulométrique de la terre utilisée ainsi que le sol corrigé montre que ses courbes sont approximativement parallèles aux courbes limites et à la courbe idéale.

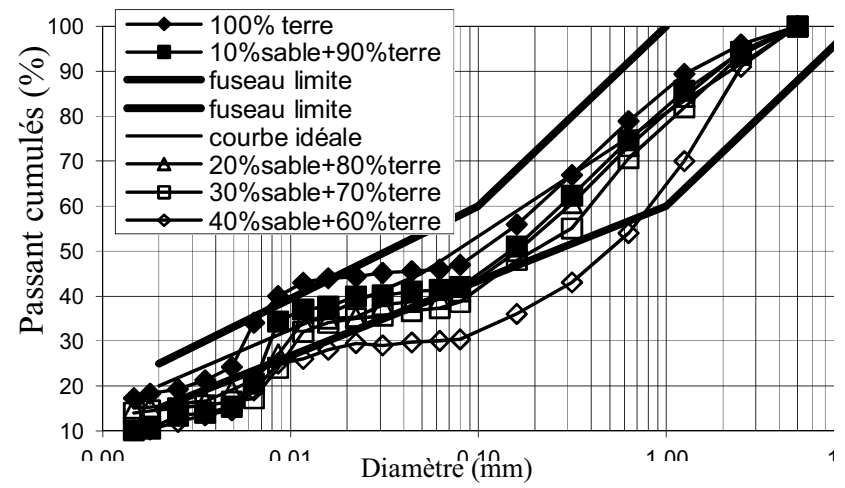

Fig.1 Courbes granulométriques des mélanges (sol, sable 1) avec la courbe idéale et le fuseau limite pour les briques de terre stabilisé.

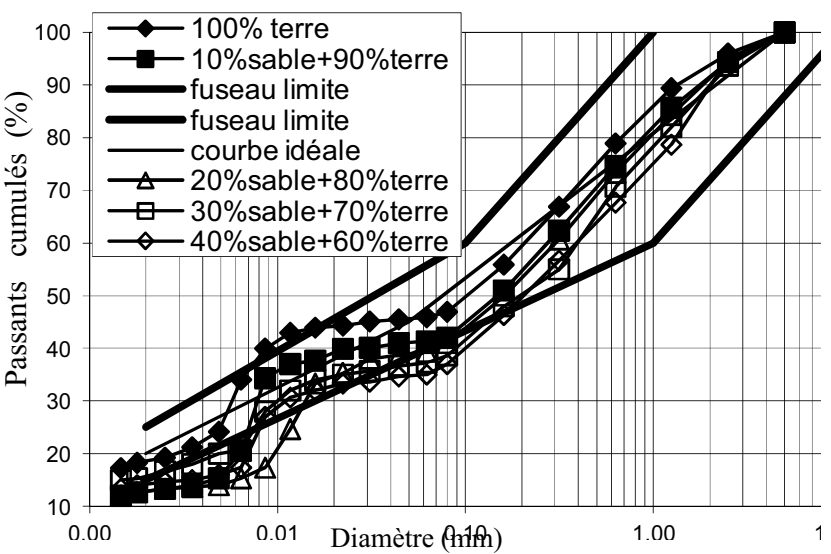

Fig.2 Courbes granulométriques des mélanges (sol, sable 2) avec la courbe idéale et le fuseau limite pour les briques de terre stabilisé.

\subsubsection{Limite d'Atterberg}

Comme pour la granularité, des études statistiques ont été faites en vue de définir les limites d'Atterberg idéales pour le béton de terre. On remarque que notre sol se situe dans la zone limite de $(25<\mathrm{WL}<50)$ et dans la zone préférable de $\mathrm{Wp}(12<\mathrm{WP}<22)$. Il a une activité moyenne $(0.75<\mathrm{Ca}<1.25)[2]$.

Tableau 2 : limites d'Atterberg

\begin{tabular}{|c|c|c|c|}
\hline $\boldsymbol{W}_{\boldsymbol{L}}$ & $\boldsymbol{W}_{\boldsymbol{P}}$ & $\boldsymbol{I P}$ & $\boldsymbol{C}_{\boldsymbol{a}}$ \\
\hline 37 & 17 & 20 & 1.08 \\
\hline
\end{tabular}




\subsubsection{Analyse minéralogique}

L'analyse minéralogique par les rayons $\mathrm{X}$ est indispensable pour différencier les sols argileux. L'analyse est effectuée au laboratoire de Boumerdes en utilisant le diffractomètre SIEMENS 500. Les résultats obtenus sont présentés dans le tableau 3, ces résultats montrent que le sol est composé de minéraux non gonflants où de gonflement négligeable (Kaolinite, Illite).

Tableau 3 : Constituants minéralogique du sol

\begin{tabular}{|c|c|c|c|}
\hline Kaolinite & Illites & Quartz & Calcite \\
\hline 45 & 40 & 5 & 10 \\
\hline
\end{tabular}

\subsubsection{Matière organique}

Pendant le traitement du sol, à 1 'eau oxygénée, nous avons constaté que la réaction terre- eau oxygénée est lente et que la matière organique a été essentiellement constituée de débris végétaux libres.

Mesure de PH Celle-ci déterminée à l'aide d'un PH mètre sur une suspension de 10 grammes du matériau sec dans 100 millilitres d'eau déminéralisé. Le résultat de l'essai nous donne un $\mathrm{PH}=7.2$, relativement neutre.

\subsubsection{Caractéristique mécaniques (essai Proctor)}

Les résultats de l'essai sont représentés dans le tableau 4

Tableau 4 : Caractéristiques mécaniques

\begin{tabular}{|c|c|c|c|c|}
\hline $\begin{array}{c}\text { Teneur } \\
\text { en eau } \\
\text { optimale } \\
\text { (T.E.O) } \\
\%(1)\end{array}$ & $\begin{array}{c}\text { Masse } \\
\text { volumique } \\
\text { maximale } \\
\text { sèche Max } \\
\gamma_{\max } \\
\left(\mathrm{kg} / \mathrm{cm}^{2}\right) \\
(2)\end{array}$ & 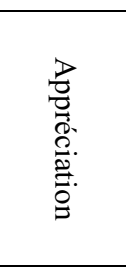 & 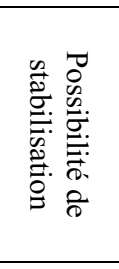 & 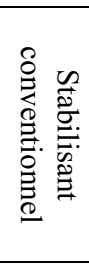 \\
\hline 9.61 & 1782 & 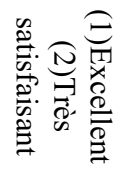 & 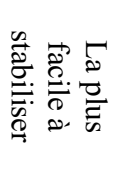 & 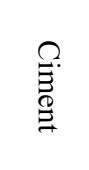 \\
\hline
\end{tabular}

D'après l'essai Procter le ciment est le stabilisant conventionnel de notre sol [6-7]

\subsection{Ciment}

Le ciment utilisé est un ciment portland composé CPJ45 fabriqué à la cimenterie d'Ain-Touta, wilaya de Batna. Afin de déterminer la réelle activité du ciment utilisé dans notre étude et selon les normes AFNOR les résultats obtenus sont donnés sur le tableau 5.

Tableau 5 : Activité du ciment

\begin{tabular}{|c|c|c|c|}
\hline Jours & $\begin{array}{c}\text { A } 7 \\
\text { jours }\end{array}$ & A 14 jours & A 28 jours \\
\hline $\begin{array}{c}\text { Résistance à la } \\
\text { compression en } \\
\text { (bars) }\end{array}$ & 343 & 392 & 461 \\
\hline
\end{tabular}

L'activité réelle du ciment est donc égale à 461 bars.

\section{Influence de la teneur en sable}

Afin d'étudier l'influence de la concentration en sable nous avons adopté cinq teneurs en sable : $0,10,20,30$ et $40 \%$ du poids du sol. Alors nous avons fabriqué pour le sol étudié une série d'éprouvettes dosées à teneurs en sable croissantes et compactées à une pression de 100 bars. La teneur en ciment est fixée à $5 \%$.

\subsection{Résistance mécanique à la compression}

Les figures 3 et 4 montrent la résistance à la compression à l'état sec et à l'état humide. Pour les deux sables nous notons une augmentation de la résistance à la compression à l'état sec et à l'état humide. Plus nous rajoutons le sable au sol plus nous augmentons la qualité d'éléments résistants les résultats similaires aux résultats obtenues par [4].

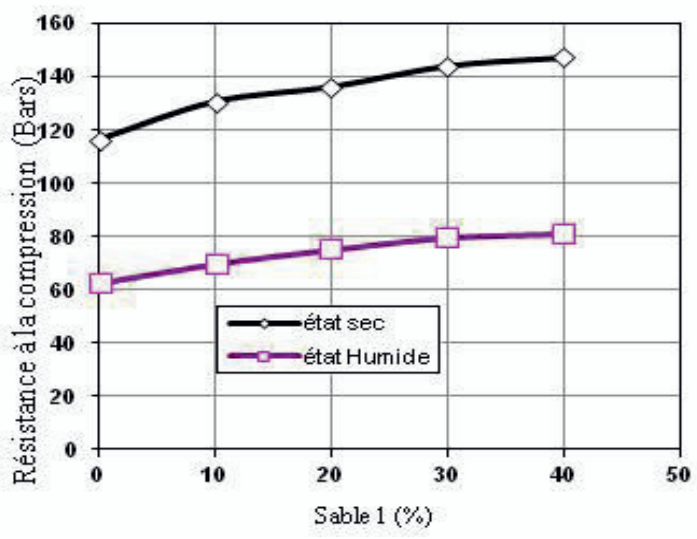

Fig. 3. Evolution de la résistance à compression à l'état sec et à l'état humide en fonction de la teneur en sable

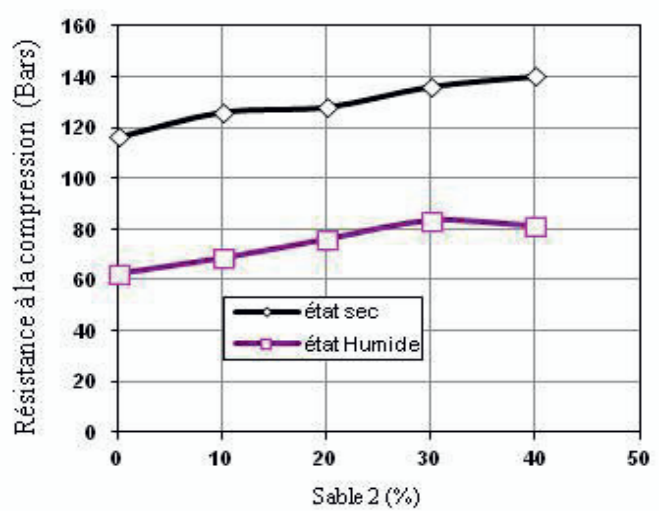

Fig. 4. Evolution de la résistance à compression à l'état sec et à l'état humide en fonction de la teneur en sable 2

\section{3-2- Coefficient de ramollissement}

La figure 5 montre que la concentration de sable 1 n'influe pas énormément sur le coefficient de 
ramollissement. Ce coefficient varie de 0.53 à 0.55 quand la teneur en sable varie de 0 à $30 \%$, par contre pour le sable 2 on constate une amélioration remarquable varie de 0.53 à 0.63 .

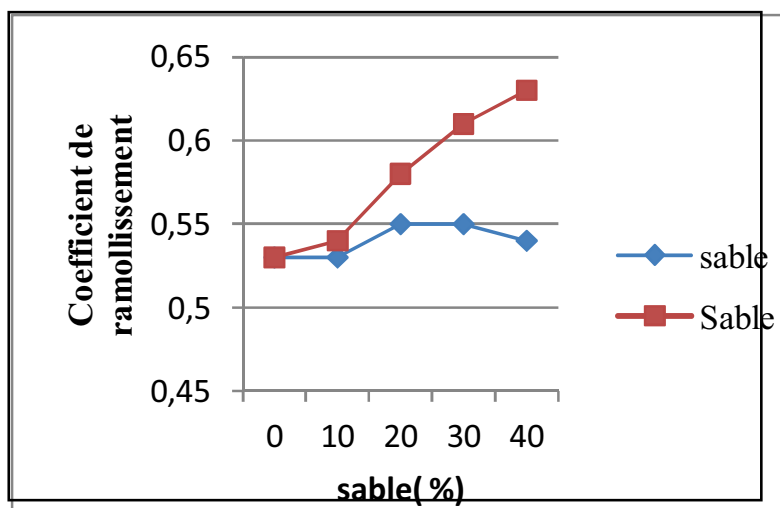

Fig. 5. Coefficient de ramollissement en fonction de la teneur en sable

\subsection{Absorption}

L'analyse de la figure 6 nous montre que la concentration de sable ne présente pas d'écart important avec celles de la terre seule, mais cette concentration a une valeur optimale qui donne une absorption minimale, cette valeur optimale correspond à $30 \%$ pour les deux type de sable.

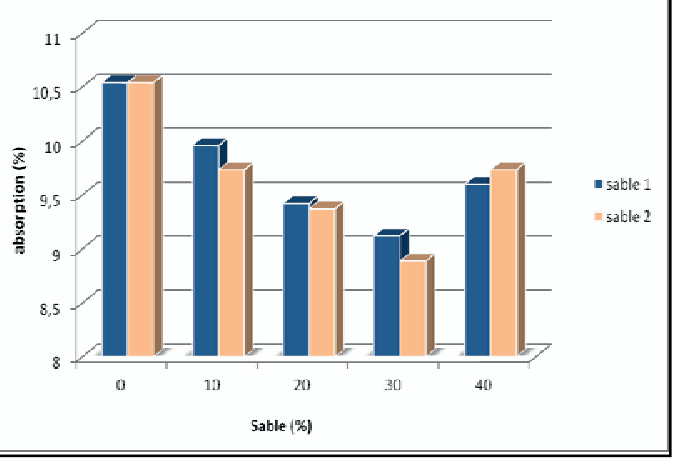

Fig. 6. Absoption totale en fonction de la teneur en sable

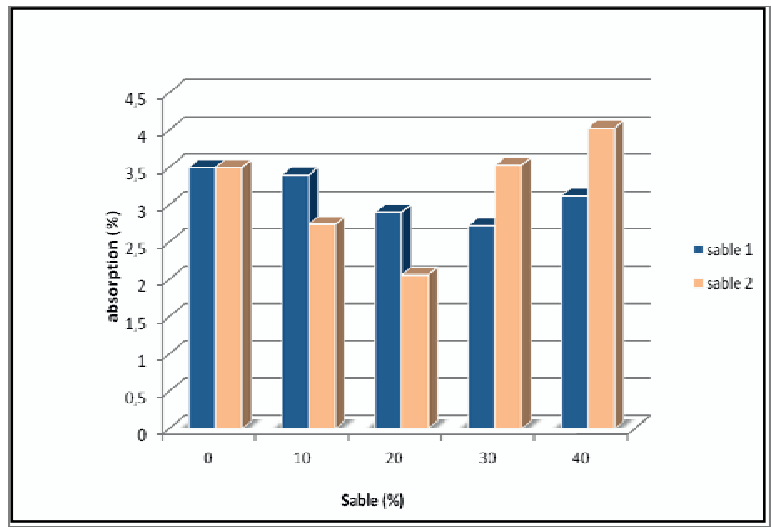

Fig. 7. Absorption cappilaire en fonction de la teneur en sable

\subsection{Durabilité (mouillage-séchage)}

Selon les normes ASTM D 559-57 [8], il consiste à faire sécher les éprouvettes jusqu'à poids constant à une température de $60^{\circ}$ à $70^{\circ} \mathrm{C}$, les éprouvettes sont pesées après le séchage puis soumises à une série de cycles comprenant :

- 5heures d'immersion au bout des quelles l'éprouvette est pesée.

- 42 heures de séchage à l'étuve à $71^{\circ} \mathrm{C}$.

La figure 8 montre que les pertes en poids après douze cycles de mouillage-séchage ne dépassent pas $5 \%$ ces pertes en poids diminuent quand la concentration en sable augmente, mais pour le sable 1, il apparaît qu'au-delà d'une concentration de $30 \%$ de sable $70 \%$ de terre, une augmentation de perte en poids et qui représente une valeur optimale, en revanche, les pertes en poids pour le sable 2 diminues avec un écart très important (de 4,30\% avec la terre seul à 1.36 avec $10 \%$ de sable, alors une diminution de $3 \%$ )

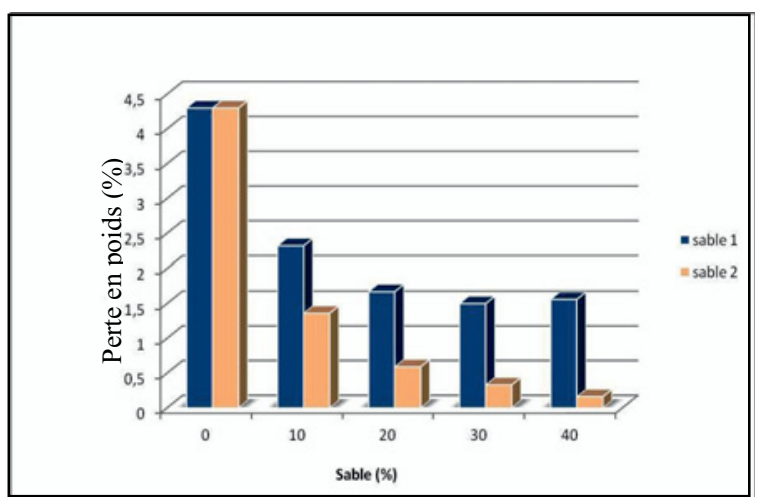

Fig. 8. Pertes en poids en fonction de la teneur en sable stabilisé

\subsection{Essai gel-dégel}

Après l'essai d'absorption, les éprouvettes sont soumises d'abord à la congélation sous une température de $-105^{\circ} \mathrm{C}$ pendant 4 heures, puis au dégel (en les immergeant dans de l'eau pendant 4 heures). Ce cycle est répété 15 fois. L'analyse de la figure 9 montre que l'accroissement de la teneur en sable n'influe pas énormément sur le comportement du matériau au gel ainsi que les deux sables présentent un optimum de $30 \%$ pour le sable 1 et

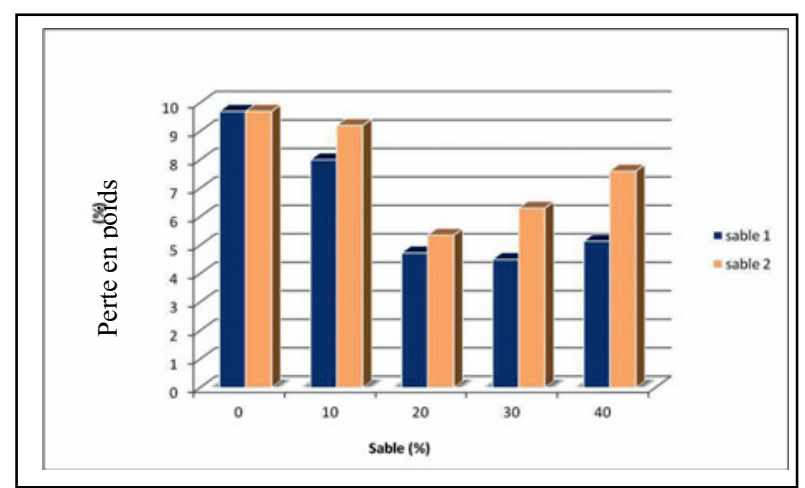

Fig.9. Pertes en poids en fonction de la teneur en sable 


\section{Conclusion}

L'étude expérimentale nous a permis de préciser un nombre de points:

- Identification des sols avant leur utilisation est nécessaire, elle pourra également conduire à des recommandations concernant leur éventuel traitement.

- La correction granulométrique peut répondre à la stabilisation. Dans ce cadre la correction granulométrique par deux types de sable, à donnée des résultats performants.

- L'augmentation de la concentration du sable améliore considérablement les résistances mécaniques de compression à l'état $\mathrm{sec}$ et à l'état humide, mais présente un optimum de $30 \%$ de sable, pour les essais de durabilité, absorption totale et capillaire. Ces résultats montrent que les éléments les plus résistants ne veulent dire pas que ce sont les plus durables.

- La correction granulométrique par le sable 2 donne des meilleures résistances mécaniques et de durabilité vis-à-vis de l'eau par rapport au sable 1 .

- Avec n'importe qu'elle teneur en sable1 et sable 2, la perte en poids lors de la durabilité ne dépasse pas $5 \%$, qui convient bien pour construire même dans les régions humides.

\section{REFERENCES}

1. Hammond, A. A., Prolongation de la durée de vie des constructions en terre sous les tropiques.

2. Guettala.A " Amélioration du béton de terre stabilisée vis à vis de l'eau", thèse de Doctorat à l'université (Biskra ,2003).

3. Ghoumari, F., Matériau en terre crue compactée: amélioration de sa durabilité à l'eau, thèse de doctorat, (INSA de Lyon, France, 1989).

4. Guettala, A. et Mezghiche, B., Vibrocompactage $d u$ matériau terre pour l'élaboration des blocs à bâtir, Revue de la 2ème congrée algérien de la route, Tome 3, p(117-125), (Décembre 1996).

5. AFNOR (1984), Recueil de Normes françaises. Bâtiment Béton et Constituants du Béton, Paris, France.

6. Houben \& Guillaud "Earth construction primer Brussels", (CRATerre , 1984).

7. Guettala, A. et Guenfoud, M., Influence des types d'argiles sur les propriétés mécaniques du béton de terre stabilisé au ciment, ANNALES $\mathrm{du}$ bâtiment et des travaux publics, $\left(\mathrm{N}^{\circ} 1\right.$, $\mathrm{pp}(15-25)$, Paris, France).

8. American Society for Testing and materials. Annual Book of ASTM standards, (V.04.01, Philadelphia). 